\title{
Correspondence
}

\section{AMANTADINE TREATMENT FOR REFRACTORY PAIN AND FATIGUE IN PATIENTS WITH MULTIPLE SCLEROSIS}

\section{To the Editor:}

Pain and fatigue are distressing symptoms of multiple sclerosis (MS). Recently several authors have shown the beneficial effects of oral amantadine hydrochloride for fatigue in MS patients. ${ }^{12.3}$ We hereby report that amantadine was effective not only for fatigue but also for chronic pain in MS.

Seven clinically definite and one probable MS patients ( 2 males and 6 females, mean age; 42.8 years), who had been suffering from chronic pain and fatigue, were studied. They had been medicated either singularly or with combinations of carbamazepine, valproate, clonazepam, maprotiline and pimozide without satisfactory response. Prior to the study, informed consent was obtained. Medications other than amantadine were not changed throughout the study, and amantadine was given $100 \mathrm{mg}$ BID or TID for at least two weeks. Clinical effects were evaluated by a daily questionnaire where each patient was asked to describe the site, quality and severity of pain/fatigue and to score the degree of pain/fatigue according to the rating scale.

Some benefits appeared within one week if amantadine was effective. Five patients showed a marked reduction of heavy and tingling pain, not neuralgic in nature, in the face and the back; however, one dropped out on eighth day because of dizziness, thirst and abnormal perspiration. Four patients showed a continuous alleviation of pain for months, but one of them had a recurrence of pain three months later, which necessitated stopping amantadine. Fatigue improved in three, and, moreover, two of them were the responders to amantadine for pain relief. We failed to find any correlation between the efficacy for pain/ fatigue and the site and quality of pain/fatigue. Neurologic signs were not influenced by amantadine.

In a double-blind placebo-controlled crossover study, Rosenberg and Appenzeller reported that amantadine was effective for fatigue in six of ten MS patients and serum levels of $\beta$-endorphin- $\beta$-lipotropin were significantly higher in responders than nonresponders. ${ }^{3}$ Their research may partly explain the mechanism of action of amantadine.

Our open study is small in number and certainly further investigation is needed, but at present we recommend amantadine for chronic refractory pain and fatigue in MS patients when conventional therapy is not effective.

Susumu CHIBA

Michiko ITO

Hiroyuki MATSUMOTO

Department of Rehabilitation and Neurology

Sapporo Medical College, Sapporo

1. The Canadian MS Research Group. A randomized controlled trial of amantadine in fatigue associated with multiple sclerosis. Can J Neurol Sci 1987; 14: 273-278.

2. Cohen RA, Fisher M. Amantadine treatment of fatigue associated with multiple sclerosis. Arch Neurol 1989; 46: 676-680.

3. Rosenberg GA, Appenzeller O. Amantadine, fatigue, and multiple sclerosis. Arch Neurol 1988; 45: 1104-1106.

\section{WEATHER AND HEALTH}

\section{To the Editor:}

Mr. Denis Bourque of the Atmospheric Environment Service of Canada and myself would like to hear from physicians across Canada who are interested in the relationship between the weather and health.

We are exploring the possibility of establishing an information network for physicians and possibly the public.

Any physician with an interest in, or information on this subject please write to one of us.

Dr.J. Bart,

Bathurst-Steeles Health Centre, 6257 Bathurst Street,

2nd Floor,

Willowdale, Ontario

$M 2 R 2 A 5$
Mr: Denis Bourque,

A.E.S.,

4905 Dufferin Street.

Downsivew, Ontario

M3H 5T4 